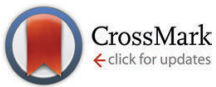

Cite this: Chem. Commun., 2017, 53,368

Received 28th October 2016 Accepted 25th November 2016

DOI: $10.1039 / c 6 c c 08671 c$

www.rsc.org/chemcomm

\section{Site-selective installation of BASHY fluorescent dyes to Annexin $V$ for targeted detection of apoptotic cells $\dagger$}

\author{
Pedro M. S. D. Cal, ${ }^{\mathrm{ab}}$ Florian Sieglitz, ${ }^{a}$ Fábio M. F. Santos, ${ }^{\mathrm{C}}$ Cátia Parente Carvalho, ${ }^{d}$ \\ Ana Guerreiro, ${ }^{a}$ Jean B. Bertoldo, ${ }^{b}$ Uwe Pischel, ${ }^{d}$ Pedro M. P. Gois ${ }^{\star c}$ and \\ Gonçalo J. L. Bernardes*ab
}

\begin{abstract}
Fluorophores are indispensable for imaging biological processes. We report the design and synthesis of azide-tagged boronic acid salicylidenehydrazone (BASHY) dyes and their use for site-selective labelling of Annexin V. The Annexin V-BASHY conjugate maintained function and fluorescence as demonstrated by the targeted detection of apoptotic cells.
\end{abstract}

Molecular imaging is a life sciences multidiscipline that reflects the analysis of biological processes in living systems at a cellular and molecular level. ${ }^{1-4}$ Of the current methods available, optical imaging is perhaps the most relevant because of its highsensitivity, the use of non-radioactive components, low cost and easy access. ${ }^{5-8}$ Therefore, it is not surprising that the past two decades have witnessed the disclosure of a large number of optical imaging probes aimed at evaluating the expression and activity of specific molecules or biological processes. ${ }^{9,10}$ Furthermore, the conjugation of optical imaging probes with diseasespecific ligands such as antibodies enables the targeted imaging of particular tissues which is of key relevance, for example, in cancer diagnosis. ${ }^{11}$ While probes with optimal fluorescence properties are often reported, the design of probes equipped with suitable handles that can be directly used in site-selective protein modification approaches is less common. This is of key importance since probe conjugation can often result in fluorescence quenching or loss of protein function.

Very recently we reported the synthesis of a new family of photostable fluorescent dyes featuring a tridentate structure

${ }^{a}$ Instituto de Medicina Molecular, Faculdade de Medicina, Universidade de Lisboa, Avenida Professor Egas Moniz, 1649-028, Lisboa, Portugal.

E-mail: gbernardes@medicina.ulisboa.pt

${ }^{b}$ Department of Chemistry, University of Cambridge, Lensfield Road,

CB21EW Cambridge, UK. E-mail: gb453@cam.ac.uk

${ }^{c}$ Research Institute for Medicines (iMed.ULisboa), Faculty of Pharmacy,

Universidade de Lisboa, Av. Prof. Gama Pinto, 1649-003, Lisboa, Portugal.

E-mail: pedrogois@ff.ul.pt

${ }^{d}$ CIQSO - Center for Research in Sustainable Chemistry and Department of Chemistry, University of Huelva, Campus de El Carmen s/n, 21071 Huelva, Spain

$\dagger$ Electronic supplementary information (ESI) available: Detailed methods and characterisation, fluorescence microscopy images. See DOI: $10.1039 / \mathrm{c} 6 \mathrm{cc} 08671 \mathrm{c}$ obtained by the modular assembly of structurally diverse boronic acids with modular Schiff base ligands. ${ }^{12}$ This unique family of boronic acid salicylidenehydrazone (BASHY) dyes show a polarity-dependent emission in the green-to-yellow spectral range, quantum yields as high as 0.6 in non-polar solvents, and a remarkable brightness similar to other widely used archetypal fluorophores such as rhodamines or cyanine dyes. ${ }^{13}$ Additionally, BASHY dyes also exhibit a highly modular structure that can be easily tuned for different applications.

Herein we show that the BASHY fluorescent core can be efficiently modified in order to incorporate a "clickable" handle to site-selectively functionalize a protein probe without compromising either the dye's fluorescent properties or the protein function (Fig. 1).

Annexin V is a protein used as an early marker of apoptosis by binding to a phospholipid membrane component, phosphatidyl serine (PS), which is translocated from the inner to the outer leaflet of the plasma membrane during apoptosis. ${ }^{14-16}$ Heterogeneous mixtures of fluorescently labelled Annexin $\mathrm{V}$ are often part of commercially available kits for detection of apoptosis. The multiple, non-site-specific incorporation of fluorescence dyes into protein biomarkers can however lead to a significant reduction in binding ability. Instead, we envisioned a two-step strategy that would allow for precise installation of a BASHY fluorescent probe at a pre-determined site within the amino acid sequence of a biomarker, in this case Annexin V, through strainpromoted azide-alkyne cycloaddition (SPAAC). ${ }^{17-20}$ Such a strategy would find broad utility not only for Annexin $\mathrm{V}$ but also for

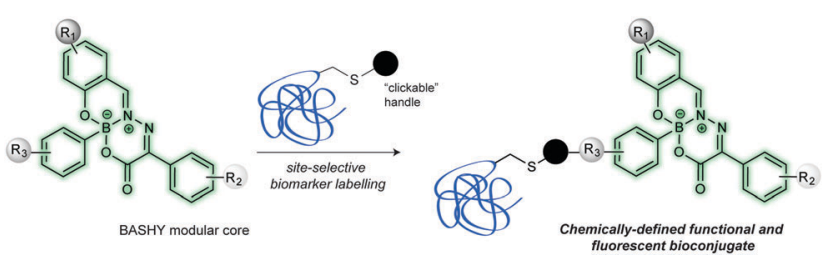

Fig. 1 Proposed site-selective modification of protein biomarkers using BASHY fluorescent dyes. 
other more sensitive protein systems where random labelling may destroy completely protein specific activity.

Considering the simple and robust synthetic route based on the modular assembly of boronic acids with Schiff-base ligands, we thought to introduce a reactive azide handle that is compatible with azide-alkyne bioorthogonal ligation reactions. Shortly, dye 3, featuring a carboxylic acid function, was readily prepared from Schiff-base 1 in 97\% isolated yield. This complex smoothly underwent TBTU mediated amidation with 2-azidoethylamine to afford compound 4 in excellent yields (Fig. 2a). Importantly, the acid (3) or azide (4)-tagged BASHY dyes retained the photophysical properties of the reported BASHY dye, ${ }^{12}$ including high quantum yields, narrow absorption and emission bands, and high molar absorption coefficients (Fig. 2b and Tables S1, S2, Fig. S1-S3, ESI $\dagger$ ). Furthermore, we studied the stability and cytotoxicity of the BASHY probe 4 . Importantly, 4 did not react with ethyleneglycol which is potentially reactive towards boronic acids (Fig. S4, ESI $\dagger$ ) and no toxic effects were observed in two different cell lines (Fig. S5, ESI $\dagger$ ).

With the azide-tagged BASHY dye 4 in hand, we decided to test its reactivity under SPAAC conditions ${ }^{21}$ using Annexin V as a model biomarker. In a first step, it was required to install the strained-alkyne counterpart on a precise site within the sequence of the protein. Annexin V displays a free, hindered cysteine (Cys) at position 316, which has been shown to be reactive using a variety of different alkylating and Michaelacceptor reagents including maleimides. ${ }^{22-24}$ Using commercially available dibenzocyclooctyne-maleimide (mal-DBCO, 5) that contains a reactive maleimide at one end and a strained-cyclooctyne ring at the other end, we tested a number of conditions for the reaction of 5 with Annexin V. We found that complete conversion could be achieved when using 25 equivalents of 5 in aqueous buffered solution (TrisHCl, $20 \mathrm{mM}$. pH 8) for 5 days at room temperature (Fig. 3 and Table S3, Fig. S6 and S7, ESI $\dagger$ ).
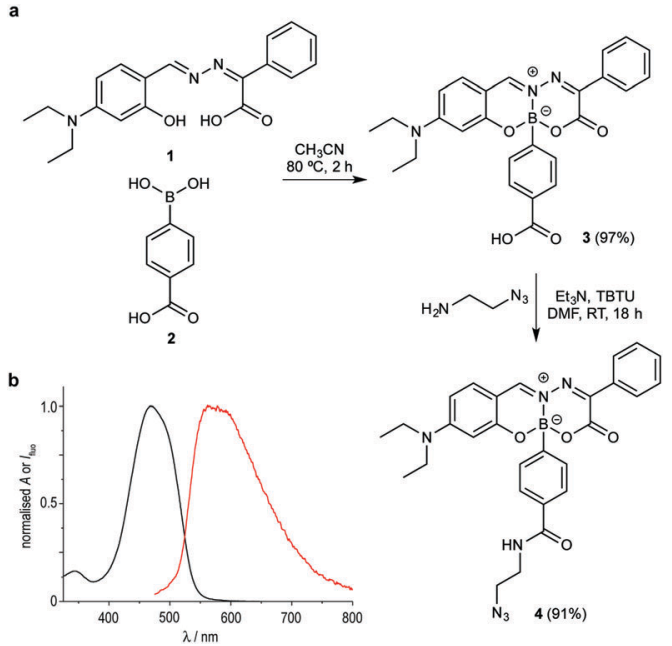

Fig. 2 (a) One pot-assembly of boronic acid-based heterocycle functionalized with a bioorthogonal handle. (b) Normalized UV/Vis absorption (black) and fluorescence (red) spectra of dye 4 in $5 \% \mathrm{DMSO} / \mathrm{NaP}_{\mathrm{i}}(50 \mathrm{mM}, \mathrm{pH}$ ). TBTU, O-(benzotriazol-1-yl)- $N, N, N^{\prime}, N^{\prime}$-tetramethyluronium tetrafluoroborate; DMF, N,N-dimethylformamide.

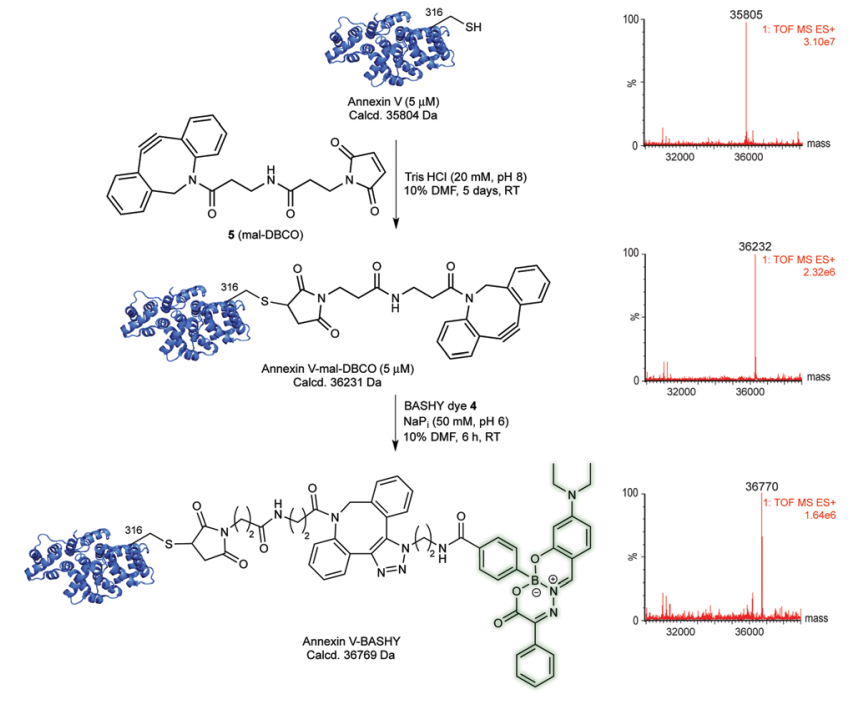

Fig. 3 Reaction of Annexin $\mathrm{V}(5.0 \mu \mathrm{M})$ with 25 equivalents of mal-DBCO 5 in Tris $\mathrm{HCl}$ buffer $(20 \mathrm{mM}, \mathrm{pH}$ 8) and $10 \% \mathrm{DMF}$, during five days at room temperature. Reaction of purified Annexin $\mathrm{V}$-mal-DBCO with 5 equivalents of BASHY dye 4 in $\mathrm{NaP}_{\mathrm{i}}$ buffer ( $50 \mathrm{mM}, \mathrm{pH}$ ) and 10\% DMF during 6 hours at room temperature. Alongside of each conjugate the deconvoluted mass spectrum is shown recording the masses for each construct.

After purification using size-exclusion chromatography, a single protein product corresponding to the mass of DBCO-tagged Annexin V was detected using liquid-chromatography massspectrometry (LC-MS) analysis (Fig. 3). Upon tryptic digest and analysis using LC-MS/MS, we confirmed that Cys316 is the primary site of modification (Fig. S8, ESI $\dagger$ ).

With the strained-alkyne moiety site-selectively installed at Cys 316 of Annexin V, we next tested the labelling SPAAC reaction using the azide-BASHY fluorescent dye 4. Labelling proceeded smoothly in sodium phosphate buffer $\left(\mathrm{NaP}_{\mathrm{i}}, 50 \mathrm{mM}, \mathrm{pH}\right.$ 6) and completion was achieved after 6 hours at room temperature (Fig. 3). Gratifyingly, and after purification, a homogenous Annexin V-BASHY construct was identified by LC-MS analysis (Fig. 3 and Fig. S9, ESI $\dagger$ ). The secondary structure protein content of the conjugates Annexin V-mal-DBCO and Annexin V-BASHY was analysed by circular dichroism (Fig. S10, ESI $\dagger$ ), showing no significant differences from unmodified Annexin V.

In order to have a basis of comparison, a commercially available azide dye (sulfo-cyanine5 azide - Cy5- $\mathrm{N}_{3}$ ) was used to label Annexin $\mathrm{V}$ under identical SPAAC conditions. This near-infrared (NIR) optical probe was chosen as a reference because it is routinely used in biological imaging including apoptosis when conjugated to Annexin V., 25-27 The site-selectively modified Annexin V-Cy5 was used as a specific positive control, since Annexin $\mathrm{V}$ is usually labelled in a non-selective manner through lysine (Lys) bioconjugation using this dye. We have also prepared an heterogeneous Annexin $\mathrm{V}$ conjugate using an NHS-ester activated fluorescein isothiocyanate isomer I (NHS-FITC). ${ }^{16}$ Both conjugates were purified to afford two fluorescent conjugates, the homogenous Annexin V-Cy5 and heterogeneous Annexin V-FITC (see Fig. S11 and S12, ESI $\dagger$ ).

The three synthesised and purified conjugates were assessed for their potential to targeted imaging of apoptotic cells using 

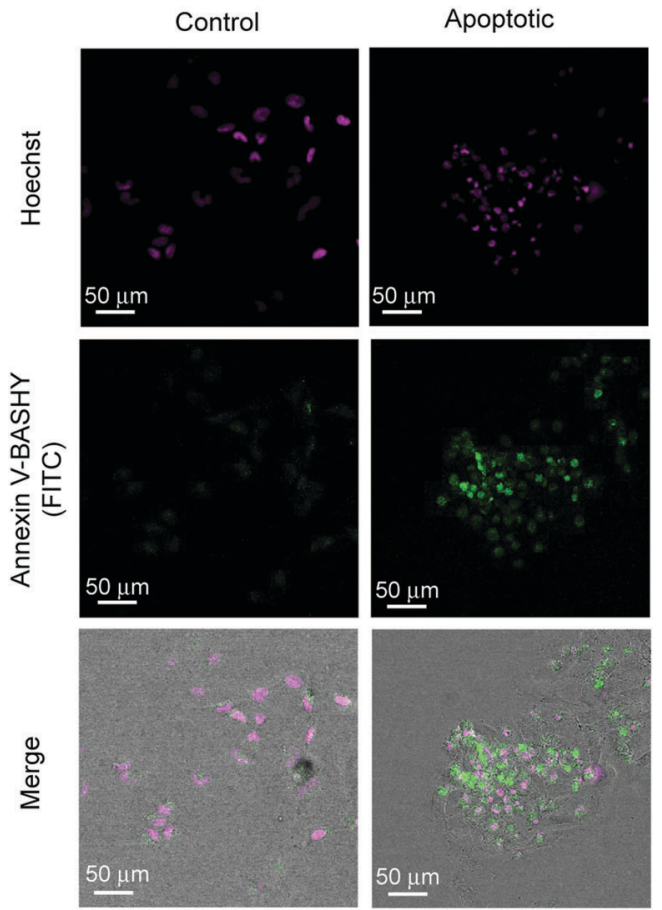

Fig. 4 Confocal microscopy images of HeLa cells treated with $1 \mu \mathrm{M}$ actinomycin $D$ for 6 hours to induce apoptosis or the vehicle control (0.1\% DMSO) followed by incubation with $1 \mu \mathrm{g} \mathrm{ml}^{-1}$ of Annexin V-BASHY for 20 min. Annexin V-BASHY (green), nuclear Hoechst staining (purple).

confocal microscopy. ${ }^{2}$ For this purpose, we used actinomycin D as an apoptosis inducer and DMSO as the negative control. ${ }^{16}$ HeLa cells were incubated with either actinomycin D or DMSO (control) for 6 hours followed by incubation with Annexin V-BASHY for 20 minutes. Finally, cells were fixed and the nuclei stained with Hoechst 33342 (Fig. 4). The specificity for PS binding of the homogenous Annexin V-Cy5 dye conjugate and the heterogenous Annexin V-FITC were also analysed using the same protocol in separate trials (see Fig. S13-S20). Gratifyingly, the Annexin V-BASHY conjugate was successfully able to target apoptotic cells with similar efficiency as both control constructs (Fig. 4). Moreover, in a blocking experiment where cells were incubated with non-fluorescent Annexin V-mal-DBCO before addition of Annexin V-BASHY, we observed a significant decrease in fluorescence which demonstrates the specificity of the BASHY-tagged biomarker (Fig. S15, ESI $\dagger$ ). These data demonstrate the utility of BASHY-protein conjugates built using site-selective protein labelling approaches for bioimaging due to their stability and non-detectable quenching or photobleaching. These properties lead to low levels of unselective fluorescence background and high signal-to-noise ratio, as demonstrated for the detection of apoptotic cells through confocal microscopy (Fig. S13-S20, ESI $\dagger$ ).

In this communication, we report for the first time the structural modification of BASHY fluorescent dyes specifically designed for the site-selective labelling of a protein biomarker. The BASHY core was simply modified with an azide-tag without altering the core photophysical properties and was then used in a two-step protocol that enabled the precise installation of the fluorescent dye at position 316 of the apoptosis biomarker Annexin V. Importantly, the fluorescence of BASHY was retained after protein labelling and the conjugate successfully targeted and detected apoptotic cells maintaining high levels of specific activity. Our strategy uses a synthetically accessible core that permits the facile introduction of "clickable" handles for subsequent precise protein fluorescent labelling. This strategy that has been successfully demonstrated for the labelling of Annexin V and detection of apoptotic cells may find broader utility when applied to protein biomarkers that are sensitive and loose their activity when randomly conjugated. Additionally, and as a future application, the reported combined synthesis and protein site-selective labelling using BASHY dyes, can be particularly useful for multimodality imaging applications, ${ }^{28-30}$ since the BASHY core can act itself as an optimal imaging agent while allowing attachment of probes used in other imaging modalities (e.g., magnetic resonance imaging (MRI), single photon emission computed tomography (SPECT) or positron emission tomography (PET)).

We thank FCT Portugal (Doctoral Fellowship, SFRH/BD/ 94779/2013 to F. M. F. S., Postdoctoral Fellowship, SFRH/ BPD/103172/2014 to P. M. S. D. C.; projects PTDC/QUI-QUI/ 118315/2010 and PTDC/BBB BQB/0506/2012; PTDC/QEQ-QOR/ 1434/2014: PTDC/SAUFAR/119389/2010; FCT Investigator to G. J. L. B. and P. M. P. G.; iMed.ULisboa grant UID/DTP/04138/ 2013), EU (Marie-Curie CIG to G. J. L. B.; Marie-Sklodowska Curie ITN ProteinConjugates to G. J. L. B. and P. M. P. G.), DFG (SI 2117/11 to F. S.), CNPq Brazil (fellowship 200456/2015-6 to J. B. B.); Ministerio de Economía y Competitividad, Madrid, Spain (grant CTQ2014-54729-C2-1-P), Junta de Andalucía (grant P12-FQM-2140) and the EPSRC (G. J. L. B.) for financial support. G. J. L. B. is a Royal Society University Research Fellow and the recipient of a European Research Council Starting Grant (TagIt).

\section{Notes and references}

1 Z. Y. Chen, Y. X. Wang, Y. Lin, J. S. Zhang, F. Yang, Q. L. Zhou and Y. Y. Liao, BioMed Res. Int., 2014, 2014, 819324.

2 B. P. Joshi and T. D. Wang, Cancers, 2010, 2, 1251-1287.

3 S. Achilefu, Chem. Rev., 2010, 110, 2575-2578.

4 A. Signore, S. J. Mather, G. Piaggio, G. Malviya and R. A. Dierckx, Chem. Rev., 2010, 110, 3112-3145.

5 M. Herranz and A. Ruibal, J. Oncol., 2012, 2012, 863747.

6 F. Leblond, S. C. Davis, P. A. Valdes and B. W. Pogue, J. Photochem. Photobiol., B, 2010, 98, 77-94.

7 V. Ntziachristos, Annu. Rev. Biomed. Eng., 2006, 8, 1-33.

8 C. McDonagh, C. S. Burke and B. D. MacCraith, Chem. Rev., 2008, 108, 400-422.

9 D. D. Nolting, M. L. Nickels, N. Guo and W. Pham, Am. J. Nucl. Med. Mol. Imaging, 2012, 2, 273-306.

10 W. Zeng, Z. Liu and W. Wang, in Molecular Imaging, ed. B. Schaller, Intech, 2012, ch. 17, DOI: 10.5772/31836.

11 R. Alford, M. Ogawa, P. L. Choyke and H. Kobayashi, Mol. BioSyst., 2009, 5, 1279-1291.

12 F. M. F. Santos, J. N. Rosa, N. R. Candeias, C. P. Carvalho, A. I. Matos, A. E. Ventura, H. F. Florindo, L. C. Silva, U. Pischel and P. M. P. Gois, Chem. - Eur. J., 2016, 22, 1631-1637.

13 L. D. Lavis and R. T. Raines, ACS Chem. Biol., 2014, 9, 855-866.

14 E. A. Schellenberger, A. J. Bogdanov, A. Petrovsky, V. Ntziachristos, R. Weissleder and L. Josephson, Neoplasia, 2003, 5, 187-192.

15 J. F. Tait, D. F. Gibson and C. Smith, Anal. Biochem., 2004, 329, 112-119. 
16 S. E. Logue, M. Elgendy and S. J. Martin, Nat. Protoc., 2009, 4, 1383-1395.

17 C. S. McKay and M. G. Finn, Chem. Biol., 2014, 2, 1075-1101.

18 J. C. Jewetta and C. R. Bertozzi, Chem. Soc. Rev., 2010, 39, 1272-1279.

19 J. M. Baskin, J. A. Prescher, S. T. Laughlin, N. J. Agard, P. V. Chang, I. A. Miller, A. Lo, J. A. Codelli and C. R. Bertozzi, Proc. Natl. Acad. Sci. U. S. A., 2007, 104, 16793-16797.

20 X. Liu, A. L. Miller, K. A. Fundora, M. J. Yaszemski and L. Lu, ACS Macro Lett., 2016, 5, 1261-1265.

21 N. J. Agard, J. A. Prescher and C. R. Bertozzi, J. Am. Chem. Soc., 2004, 126, 15046-15047.

22 X. Li, J. M. Link, S. Stekhova, K. J. Yagle, C. Smith, K. A. Krohn and J. F. Tait, Bioconjugate Chem., 2008, 19, 1684-1688.

23 A. Perreault, J. C. Knight, M. Wang, J. Way and F. Wuest, Amino Acids, $2016,48,65-74$.
24 P. Yu, I. Strug, T. R. Cafarella, B. A. Seaton and A. Krantz, Org. Biomol. Chem., 2012, 10, 4500-4504.

25 Z. Medarova, S. Bonner-Weir, M. Lipes and A. Moore, Diabetes, 2005, $\mathbf{5 4}, 1780-1788$.

26 V. Ntziachristos, E. A. Schellenberger, J. Ripoll, D. Yessayan, E. Graves, A. Bogdanov, Jr., L. Josephson and R. Weissleder, Proc. Natl. Acad. Sci. U. S. A., 2004, 101, 12294-12299.

27 A. Goel, F. Fuerst, E. Hotchkiss and C. R. Boland, Cancer Biol. Ther., 2014, 5, 529-535.

28 C. Perez-Medina, D. Abdel-Atti, Y. Zhang, V. A. Longo, C. P. Irwin, T. Binderup, J. Ruiz-Cabello, Z. A. Fayad, J. S. Lewis, W. J. Mulder and T. Reiner, J. Nucl. Med., 2014, 55, 1706-1711.

29 L. Sun, J. Ding, W. Xing, Y. Gai, J. Sheng and D. Zeng, Bioconjugate Chem., 2016, 27, 1200-1204.

30 E. A. Rodriguez, Y. Wang, J. L. Crisp, D. R. Vera, R. Y. Tsien and R. Ting, Bioconjugate Chem., 2016, 27, 1390-1399. 